\title{
Invasive bacterial diseases in northern Canada, 1999 to 2018
}

\author{
Grace Huang1', Irene Martin², Raymond S Tsang², Walter H Demczuk², Gregory J Tyrrell', \\ Y Anita Li*, Catherine Dickson'1, Francesca Reyes-Domingo', Susan G Squires ${ }^{1}$
}

\begin{abstract}
Background: The International Circumpolar Surveillance (ICS) program conducts surveillance on five invasive bacterial diseases: pneumococcal disease (IPD), group A streptococcus (iGAS), Haemophilus influenzae (Hi), meningococcal disease (IMD) and group B streptococcus (GBS). Invasive bacterial diseases have a higher burden of disease in northern populations than the rest of Canada.
\end{abstract}

Methods: To describe the epidemiology of invasive bacterial diseases in northern Canada from 1999 to 2018, data for IPD, iGAS, Hi, IMD and GBS were extracted from the ICS program and the Canadian Notifiable Diseases Surveillance System (CNDSS) and analyzed.

Results: The annualized incidence rates for IPD, iGAS, Hi, GBS and IMD were 23.3, 10.5, 8.9, 1.9 and 1.1 per 100,000 population, respectively. The incidence of IPD, iGAS and Hi serotype $b$ were 2.8, 3.2 and 8.8 times higher, respectively, in northern Canada than in the rest of Canada. Rates of disease decreased statistically significantly for IPD $(\beta=-0.02)$ and increased statistically for iGAS $(\beta=0.08)$ and Hi serotype a $(\beta=0.04)$ during the study period. In Northern Canada, the annualized incidence rates for IPD, iGAS and Hi were statistically higher for Indigenous residents than for non-Indigenous residents. The highest incidence rates were among the very young and older age groups.

Conclusion: Invasive bacterial diseases represent a high burden of disease in Canada's northern populations. Indigenous peoples, children and seniors are particularly at risk.
This work is licensed under a Creative Commons Attribution 4.0 International License.

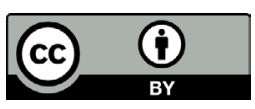

Affiliations

${ }^{1}$ Infectious Disease Programs Branch, Public Health Agency of Canada, Ottawa, ON

${ }^{2}$ National Microbiology Laboratory, Public Health Agency of Canada, Winnipeg, MB

${ }^{3}$ Department of Laboratory Medicine and Pathology, University of Alberta, Edmonton, $A B$

\section{*Correspondence: \\ y.anita.li@phac-aspc.gc.ca}

Suggested citation: Huang G, Martin I, Tsang RS, Demczuk WH, Tyrrell GJ, Li YA, Dickson C, Reyes-Domingo F, Squires SG. Invasive bacterial diseases in northern Canada, 1999 to 2018. Can Commun Dis Rep

2021;47(11):491-9. https://doi.org/10.14745/ccdr.v47i11a09

Keywords: Indigenous health, surveillance, Haemophilus influenzae, streptococcus, meningococcal disease, pneumococcal disease, vaccine, epidemiology

\section{Introduction}

The International Circumpolar Surveillance (ICS) program is a population-based invasive bacterial disease surveillance network of countries with circumpolar regions (1). The ICS program conducts surveillance on invasive bacterial diseases caused by Streptococcus pneumoniae (invasive pneumococcal disease, IPD), Haemophilus influenzae (invasive Haemophilus influenzae, $\mathrm{Hi}$ ), Streptococcus pyogenes (invasive group A streptococcus, iGAS), Neisseria meningitidis (invasive meningococcal disease, IMD) and Streptococcus agalactiae (invasive group B streptococcus, GBS). Disease rates caused by these pathogens are elevated among the Indigenous peoples in countries with Arctic regions (2-7).
The ICS program started monitoring IPD in Canada in 1999 and expanded to monitoring the other four diseases in 2000. Six Canadian regions participate in the ICS program: Nunavut, the Northwest Territories, Yukon, northern Labrador and two northern Québec regions, Nunavik and Cree Territory. The ICS program also includes two reference laboratories, the National Microbiology Laboratory (NML) and the Laboratoire de santé publique du Québec (LSPQ), and a network of regional laboratories located across Canada that serves the northern regions. Up until 2009, the National Centre for Streptococcus (NCS) participated in the ICS program as a reference laboratory. 
In 2018, the Canadian ICS population was estimated to be 168,090 , accounting for $0.45 \%$ of the total national intercensal population of 37 million. While Indigenous people account for $4.9 \%$ of the total Canadian population, they represent approximately $60 \%$ of the northern Canadian population (8).

The National Advisory Committee on Immunization (NACl) provides national vaccine recommendations for IPD, $\mathrm{HI}$ and IMD; however, adoption of these recommendations are the responsibility of the provincial and territorial jurisdictions. The $H$. influenzae serotype b (Hib) vaccine, the meningococcal $C$ conjugate $(\mathrm{MenC})$ vaccine for infants and the Men-C-ACYW vaccine for early adolescents have been part of routine childhood immunization programs since 1997, 2007 and 2011, respectively (9). As part of the routine childhood immunization schedule, all six northern regions implemented the 7-valent pneumococcal conjugate vaccine (PCV7) for children younger than two years of age between 2002 and 2007, replacing PCV7 with the 10-valent pneumococcal conjugate vaccine (PCV10) by 2010 (10) and subsequently with the 13-valent pneumococcal conjugate vaccine (PCV13) by 2011 (according to a survey sent to ICS invasive bacterial diseases working group members). In mid-2018, Cree Territory and Nunavik switched to a four-dose PCV10 and PCV13 $(3+1)$ vaccination schedule (personal communication, ICS invasive bacterial diseases working group members, 2019). NACl recommends that the 23-valent pneumococcal polysaccharide vaccine (PPV23) be given to those aged two years or older with high risk of IPD, and adults 65 years and older (11). High-risk children and adults include those with certain chronic diseases, immunocompromising conditions or functional or anatomic asplenia (11). The Canadian Immunization Guide recommendations for PPV23 have been implemented in all six regions, with minor differences in the age of vaccine administration in some regions (11-17).

The objective of this report is to describe the epidemiology of invasive bacterial diseases in northern Canada over the first 20 years of the ICS program (1999-2018) and compare their incidence rates to the rest of Canada. This report includes ICS data that have been previously published (1999-2013) $(5,7)$. These data were included in this paper to provide a comprehensive analysis of invasive bacterial diseases over the first 20 years of the ICS program.

\section{Methods}

\section{Epidemiological data}

Data were extracted from the ICS program (1999-2018) and the Canadian Notifiable Diseases Surveillance System (CNDSS, 2000-2018). CNDSS data from before 2000 were not available. CNDSS data for invasive Hi non-serotype $b$ were not included as only certain provinces and territories have reported these for certain years. CNDSS data for GBS were also not included because only GBS of newborns are nationally reported whereas ICS conducts surveillance on all GBS cases. Cases meeting the national case definition were included (18). Because there is no national case definition for general population GBS, the ICS case definition extends the national case definition for GBS of the newborn to individuals of all ages in northern Canada. Data include non-nominal demographic information, clinical information, severity, outcomes, underlying conditions and immunization history.

\section{Laboratory data}

S. pneumoniae isolates were serotyped using the Quellung reaction with pool, group, type and factor commercial antisera (Statens Serum Institut, Copenhagen, Denmark) (19). $S$. pyogenes isolates were identified using $\beta$-hemolysis on sheep blood agar, bacitracin susceptibility and pyrrolidonyl arylamidase test. M serotyping of invasive S. pyogenes isolates from 1999 to September 2006 was performed using a serological typing protocol described by Tyrrell et al. (20). From October 2006 to 2018, molecular emm typing of invasive $S$. pyogenes isolates was performed using polymerase chain reaction (PCR) tests and DNA sequencing according to the Centers for Disease Control and Prevention (CDC) protocol; the CDC invasive S. pyogenes emm sequence database was searched for designation of emm type using the basic local alignment search tool (BLAST) $(21,22)$. $S$. agalactiae isolates were serotyped by latex agglutination (SSI Diagnostica; Statens Serum Institute, Copenhagen, Denmark) as described by Slotved et al. (23). H. influenzae was identified using Gram stain morphology and standard biochemical tests (24). The bacterial slide agglutination test, using commercial antisera (Difco, Becton Dickinson, Mississauga, Ontario), was used for serotyping, with results confirmed using PCR (25). N. meningitidis was identified by standard biochemical tests with serogrouping determined by a bacterial agglutination tests using in-house-produced antisera against the 12 different serogroups (26).

Phenotypic antimicrobial susceptibilities for the Canadian isolates were determined using Sensititre STP6F micro-broth dilution panels (Thermo Fisher, United States). Resistant, intermediate or susceptible interpretations of minimum inhibitory concentration for erythromycin, clindamycin, penicillin, cefepime, cefotaxime, ceftriaxone, meropenem, trimethoprim/sulfamethoxazole and tetracycline were determined using Clinical Laboratory Standards Institute guidelines (27).

\section{Population data}

Population estimates were based on the final postcensal estimates for July 1, 2016 (8). The Indigenous populations for northern Canada were estimated using the 1996, 2001, 2006, 2011 and 2016 Census (8,28-31). The proportion of the Indigenous population for a given census year was used to estimate the Indigenous population for the years until the next census.

\section{Analysis}

The direct method was used to calculate age-standardized rates by multiplying the age-specific rates by the 2011 general 
Canadian population weights (32). Confidence intervals (95\% $\mathrm{Cls}$ ) of age-standardized rates were calculated using the method based on the gamma distribution (33). Two-tailed Fisher's exact and chi-square tests were used to compare proportions. Poisson and negative binomial regression were used to estimate disease trends. The exact Poisson test was used to compare rates. Statistical significance was set at $p<0.05$.

Analyses were conducted using Excel 2016 (Microsoft Corp., Redmond, Washington, United States), SAS Enterprise Guide 7.1 (SAS Institute Inc., Cary, North Carolina, United States) and $R$ version 3.6.2 (R Foundation for Statistical Computing, Vienna, Austria).

Incidence rates for neonatal GBS cases were not calculated because annual live birth estimates for the northern regions were not available for this report. Age-standardized rates by sex for IMD and GBS were not calculated due to small numbers. GBS is not reportable in the province of Québec, and therefore Cree Territory and Nunavik are not included in the analyses.

\section{Results}

\section{Overview}

From 1999 to 2018, a total of 692 cases of IPD were reported in northern Canada. From 2000 to 2018, a total of 311 cases of iGAS, 258 cases of $\mathrm{Hi}, 44$ cases of GBS and 31 cases of IMD were reported in the same region. Demographic information for each disease is presented in Table 1, and the overall incidence for each disease from 1999 to 2018 is presented in Figure 1.

\section{Invasive pneumococcal disease}

The regression analyses found a significant overall downward trend in IPD incidence rate (per 100,000 population) over time $(\beta=-0.02 ; p=0.01)$, but there was a sharp $92 \%$ increase in the number of cases reported from 2017 to 2018. The age-standardized incidence rates for males $(28.26$; Cl: 25.30-31.53) and females (23.86; Cl: 20.77-27.36) did not differ significantly. The incidence rate (per 100,000 population)
Figure 1: Overall crude incidence rates of invasive bacterial diseases in the ICS regions by disease and year, 1999-2018

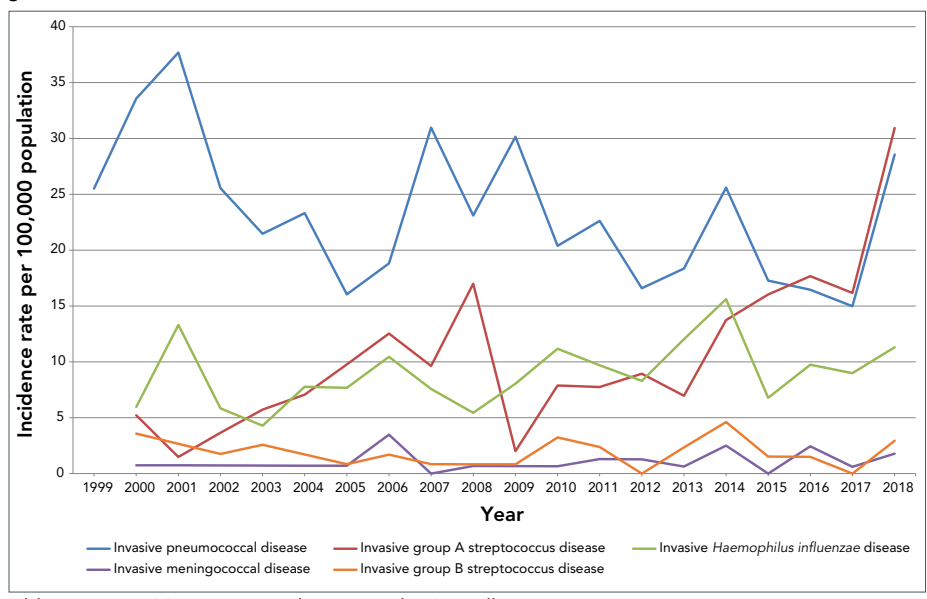

Abbreviation: ICS, International Circumpolar Surveillance

was highest among infants younger than one year old (146.78;

Cl: 117.59-183.23), children one to four years old (49.80;

$\mathrm{Cl}: 41.13-60.29)$ and adults 60 years and older (51.07;

$\mathrm{Cl}$ : 43.22-60.32). The difference in the average annual incidence rates between those of Indigenous and non-Indigenous origin was significant in northern regions (Table 1). The age-standardized incidence rate of IPD in northern regions was 2.8 times higher than the rest of Canada (Table 2).

PCV7 serotypes $(\beta=-0.19 ; \beta<0.0001)$ and PCV10-specific serotypes $(\beta=-0.01 ; p<0.0001)$ have decreased significantly from 1999 to 2018 (Figure 2). PPV23-specific serotypes $(\beta=0.06 ; p<0.0001)$ and non-vaccine serotypes have increased significantly from 1999 to $2018(\beta=0.04 ; \beta<0.05)$. There were no statistically significant changes to the PCV13-specific serotypes from 1999 to 2018 . Of the cases with available serotype data collected between 1999 and 2010, the most common were serotype $1(18 \%), 8(11 \%)$ and $14(7 \%)$. After 2010 , the most common serotypes were $7 \mathrm{~F}(12 \%), 22 \mathrm{~F}(9 \%), 10 \mathrm{~A}(9 \%)$ and 9N (8\%).

Table 1: Characterization of disease in northern Canada by crude incidence rate, demographics, fatality and hospitalization, 1999/2000-2018

\begin{tabular}{|c|c|c|c|c|c|c|c|c|c|c|c|c|c|c|c|}
\hline \multirow{3}{*}{ Disease } & \multirow{3}{*}{$\mathbf{N}$} & \multirow{3}{*}{$\begin{array}{c}\text { Crude } \\
\text { incidence } \\
\text { rate per } \\
100,000 \\
\text { population }\end{array}$} & \multirow{3}{*}{$\begin{array}{c}\text { Median } \\
\text { age, } \\
\text { years }\end{array}$} & \multirow{3}{*}{$\begin{array}{l}\text { Age } \\
\text { range }\end{array}$} & \multicolumn{3}{|c|}{$\begin{array}{l}\text { Annualized incidence rate per } \\
100,000 \text { population by ethnicity }\end{array}$} & \multicolumn{4}{|c|}{ Hospitalization } & \multicolumn{4}{|c|}{ Fatality $^{a}$} \\
\hline & & & & & \multirow{2}{*}{$\begin{array}{l}\text { Indigenous } \\
\text { ethnicity }\end{array}$} & \multirow{2}{*}{$\begin{array}{l}\text { Non- } \\
\text { Indigenous } \\
\text { ethnicity }\end{array}$} & \multirow{2}{*}{$\begin{array}{l}\text { Difference } \\
\text { between } \\
\text { ethnicities, } \\
\text { p-value }\end{array}$} & \multicolumn{2}{|c|}{ Cases } & \multicolumn{2}{|c|}{ Incicence rate ratio } & \multicolumn{2}{|c|}{ Death } & \multicolumn{2}{|c|}{ Fatality rate ratio } \\
\hline & & & & & & & & $\mathbf{N}$ & $\%$ & Male:female & $p$-value & $\mathbf{N}$ & $\%$ & Male:female & $p$-value \\
\hline IPD & 692 & 23.3 & 37 & $0-98$ & 31.3 & 7.0 & $<0.0001^{b}$ & 585 & 84.5 & 1.3 & $0.006^{b}$ & 59 & 8.5 & 1.3 & 0.3 \\
\hline iGAS & 311 & 10.5 & 50 & $0-98$ & 14.8 & 2.7 & $<0.0001^{b}$ & 273 & 87.8 & 1.3 & $0.03^{b}$ & 30 & 9.6 & 1.2 & 0.6 \\
\hline $\mathrm{Hi}$ & 258 & 8.9 & 1 & $0-93$ & 13.1 & 0.9 & $<0.0001^{b}$ & 222 & 86.0 & 1.1 & 0.5 & 19 & 7.4 & 1.6 & 0.3 \\
\hline GBS & 44 & 1.9 & 32.5 & $0-88$ & 1.8 & 1.3 & 0.3 & 41 & 93.2 & 0.9 & 0.7 & 0 & 0 & & $-c$ \\
\hline IMD & 31 & 1.1 & 1 & $0-56$ & 1.4 & 0.6 & 0.06 & 28 & 90.3 & 0.7 & 0.4 & 4 & 12.9 & All male & 0.06 \\
\hline
\end{tabular}

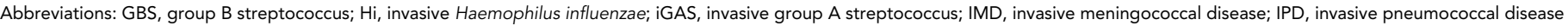
a Fatality is defined as death during the individual's illness

b Statistically significant

No deaths were reported for either males or females 
Table 2: Age-standardized incidence rates (per 100,000 population) of invasive bacterial diseases in Canada, by disease, region and year, 2000/2001-2018

\begin{tabular}{|c|c|c|c|c|}
\hline \multirow{3}{*}{ Disease } & \multicolumn{4}{|c|}{ Age-standardized incidence rates } \\
\hline & \multicolumn{2}{|c|}{ Northern regions } & \multicolumn{2}{|c|}{ Rest of Canada } \\
\hline & $95 \%$ & $\mathrm{Cl}$ & $95 \%$ & $\mathrm{Cl}$ \\
\hline $\mathrm{IPD}^{\mathrm{b}}$ & 25.68 & $23.45-28.09^{c}$ & 9.13 & $9.05-9.21^{c}$ \\
\hline iGAS & 14.16 & $12.31-15.86^{c}$ & 4.45 & $4.40-4.50^{\circ}$ \\
\hline $\mathrm{Hib}^{d}$ & 0.70 & $0.45-1.13^{c}$ & 0.08 & $0.08-0.09^{c}$ \\
\hline IMD & 0.75 & $0.51-1.17$ & 0.52 & $0.51-0.54$ \\
\hline
\end{tabular}

Abbreviations: $\mathrm{Cl}$, confidence interval; Hib, Haemophilus influenzae serotype b; ICS, International Circumpolar Surveillance; iGAS, invasive group A streptococcus; IMD, invasive meningococcal disease; IPD, invasive pneumococcal disease

a One case of IPD and one case of iGAS with missing age were excluded from the ICS incidence rate calculation

${ }^{b}$ Age-standardized incidence rates for IPD do not include data for 1999-2000, when data were either not available or not reported by all provinces

c As the Cls are not overlapped, the age-standardized incidence rates for IPD, iGAS and Hib are significantly different between northern regions and the rest of Canada

${ }^{d}$ Comparisons of age-standardized incidence rates for Haemophilus influenzae serotype non-b ( $a-f$ and non-typeable serotypes) between northern Canada and the rest of Canada were not performed as these data are not available for all provinces

Figure 2: Distribution of invasive pneumococcal disease serotypes in northern Canada, by year and by vaccine serotype $^{a}$, 1999-2018

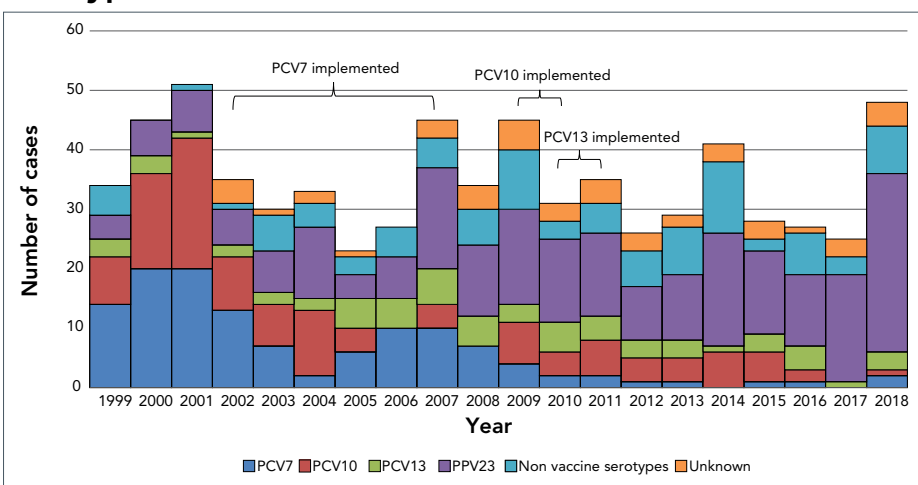

Abbreviations: PCV7, 7-valent pneumococcal conjugate vaccine; PCV10, 10-valent pneumococcal conjugate vaccine; PCV13, 13-valent pneumococcal conjugate vaccine; PPV23, 23-valent pneumococcal polysaccharide vaccine

a For the purposes of this graph, PCV7 includes all serotypes covered by the PCV7 vaccine, PCV10 includes the three additional serotypes not covered by the PCV7 vaccine, PCV13 includes the three additional serotypes not covered by the PCV10 vaccine, and PPV23 includes all serotypes not covered by any of the conjugate vaccines

All ICS regions had implemented the PCV13 vaccine schedule by January 1, 2011. Thirty-four cases that occurred after PCV13 implementation were age-appropriately vaccinated with at least one dose and serotyped. Individuals were age-appropriately vaccinated based on the minimum three-dose national recommendation. Of these 34 cases, three cases (9\%) had vaccine-breakthrough disease. Breakthrough disease occurs if the serotype of the case is any of the serotypes covered by the vaccine administered. Two of these cases were serotype 19A and one was serotype 3 . Of the 55 PPV23-vaccinated and serotyped cases, 46 cases ( $84 \%$ ) had vaccine-breakthrough disease.

Of the 670 cases with information on clinical manifestation, bacteremia $(n=577 ; 86.1 \%)$ and pneumonia $(n=442 ; 66.0 \%)$ were the most commonly reported manifestations. Of the 692 IPD cases, 585 (85\%) were hospitalized.
Of the 649 cases with information on fatality, 59 were fatal (9.1\%).The majority of the fatal cases were 60 years and older $(n=24 ; 41 \%)$, followed by those aged 40 to 59 years $(n=20 ; 34 \%)$. Individuals in these two age groups had significantly higher risk of fatality than those in younger age groups (case fatality ratio $[C F R]=14.2 \%$ vs. $4.4 \% ; p<0.0001)$. The CFR did not vary between Indigenous and non-Indigenous people (9.5\% vs. $6.1 \%$; $p=0.31$ ). Of the 53 fatal cases with serotype information, $45 \%$ were PPV23-specific serotypes not covered by PCV13. The top six reported serotypes were $20,10 \mathrm{~A}, 15 \mathrm{C}, 22 \mathrm{~F}, 15 \mathrm{~A}$ and 3.

When fatality and hospitalization rates were compared for males and females, hospitalization was significantly higher for males (Table 1).

Antimicrobial susceptibility was examined for the IPD isolates with available data (Table 3).

\section{Table 3: Proportion of antibiotic-susceptible IPD and} iGAS isolates, 1999/2000-2018

\begin{tabular}{|l|r|r|}
\multicolumn{1}{|c|}{ Antibiotic } & $\begin{array}{c}\text { Proportion of } \\
\text { susceptible IPD } \\
\text { isolates, \% }\end{array}$ & $\begin{array}{c}\text { Proportion of } \\
\text { susceptible iGAS } \\
\text { isolates, \% }\end{array}$ \\
\hline Ampicillin & 100 & 100 \\
\hline Cefotaxime & 100 & 100 \\
\hline Ceftriaxone & 98.3 & 100 \\
\hline Cefuroxime & 100 & N/A \\
\hline Chloramphenicol & 99.8 & 98.1 \\
\hline Clindamycin & 97.3 & 90.0 \\
\hline Erythromycin & 92.4 & 81.4 \\
\hline Levofloxacin & 99.8 & 98.9 \\
\hline Ofloxacin & 99.4 & 100 \\
\hline Oxacillin & 97.6 & 100 \\
\hline Penicillin & 96.1 & 100 \\
\hline Rifampin & 100 & 100 \\
\hline Tetracycline & 93.3 & 100 \\
\hline Sulfamethoxazole/ & 92.9 & 76.2 \\
\hline Trimethoprim & 100 & 100 \\
\hline Vancomycin & & \\
\hline
\end{tabular}

Abbreviations: iGAS, invasive group A streptococcus; IPD, invasive pneumococcal disease a The proportion of antimicrobial susceptible isolates for invasive Haemophilus influenzae (Hi), invasive meningococcal disease (IMD) and group B streptococcus (GBS) were not included in the analyses due to the small case counts for a number of the antibiotics tested

\section{Invasive group A streptococcal disease}

The incidence rate $(\beta=0.08 ; p<0.0001)$ of iGAS (per 100,000 population) increased significantly from 2000 to 2018 . The age-standardized incidence rates for males $(16.07 ; \mathrm{Cl}$ : 13.65-18.84) and females (11.83; Cl: 9.58-14.52) did not differ significantly. The incidence rate (per 100,000 population) was highest for infants younger than one year old (51.25; Cl: 34.90-75.27) and adults 60 years and older (36.64; $\mathrm{Cl}: 30.00-44.75)$. The difference in the average annual incidence rates between those of Indigenous and non-Indigenous 
origin was significant in northern regions (Table 1). The agestandardized incidence rate of iGAS in northern regions was 3.2 times higher than the rest of Canada (Table 2).

Of the 311 iGAS cases, 249 were serotyped. The most common emm types were emm1 with 28 cases (11.2\%), emm83 with 17 cases $(6.8 \%)$, emm3 with 15 cases $(6.0 \%)$ and emm11 and emm59 with 14 cases each (5.6\%) (Figure 3). The predominant emm type varied across the years, and while emm 1 was circulating most years, it was not always the most common strain in a given year. The most common strain in 2017 and 2018 was emm11.

Figure 3: Distribution of iGAS cases by emm type in northern Canada, 2014-2018

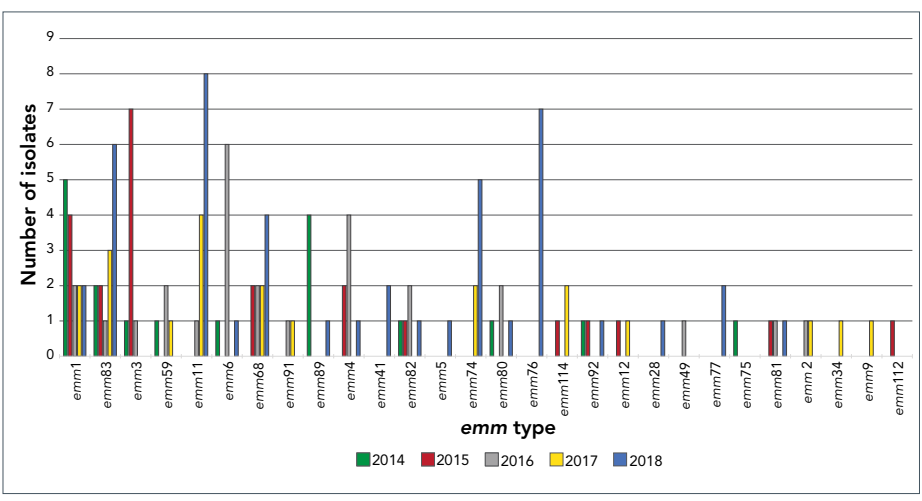

Abbreviation: iGAS, invasive group A streptococcus

Of the 307 cases with information on clinical manifestation, bacteremia $(n=196 ; 63.8 \%)$ and cellulitis $(n=96 ; 31.3 \%)$ were the most commonly reported manifestations, and 273 (88.9\%) cases were hospitalized. Of the 296 cases with outcome information, 30 deaths (CFR: 10\%) were reported. When fatality and hospitalization rates were compared for males and females, hospitalization was significantly higher for males (Table 1). The emm types with the highest fatality ratios were emm87 (50.0\%) and emm2 (50.0\%), followed by emm3 (33.3\%) and emm75 (33.3\%).

Antimicrobial susceptibility was examined for the iGAS isolates with available data (Table 3).

\section{Invasive Haemophilus influenzae disease}

There was no significant change in the $(\beta=0.02 ; p=0.06)$ incidence rate of $\mathrm{Hi}$ (per 100,000 population) from 2000 to 2018. The age-standardized incidence rates for males (7.84; Cl: 6.44-9.54) and females (6.85; Cl: 5.44-8.63) did not differ significantly. The incidence rate (per 100,000 population) was highest among infants younger than one year old (195.15; Cl: 160.29-237.59), children one to four years old (39.90; Cl: 23.05-49.67) and adults 60 years and older (9.54; $\mathrm{Cl}$ : 6.45-14.12). The difference in the average annual incidence rates between those of Indigenous and non-Indigenous origin was significant in northern regions (Table 1).
Hi serotype a (Hia) accounted for $60.5 \%$ of cases $(n=156)$, nontypeable strains accounted for $17.8 \%(n=46)$, Hib accounted for $11.2 \%(n=29)$, serotype $f$ accounted for $4.7 \%(n=12)$, serotype c and $d$ accounted for $1.6 \%$ each $(n=4)$, serotype e accounted for $0.4 \%(n=1)$ and $2.7 \%(n=6)$ had unknown serotype (Figure 4$)$. Rates of Hia increased significantly from 2000 to 2018 ( $\beta=0.04$; $p=0.01)$, and $69.2 \%(n=108)$ of Hia cases were in children up to two years old. The age-standardized incidence rate of Hib in northern regions was 8.8 times higher than the rest of Canada (Table 2).

\section{Figure 4: Distribution of invasive Haemophilus influenzae serotypes in northern Canada by year, 2000-2018}

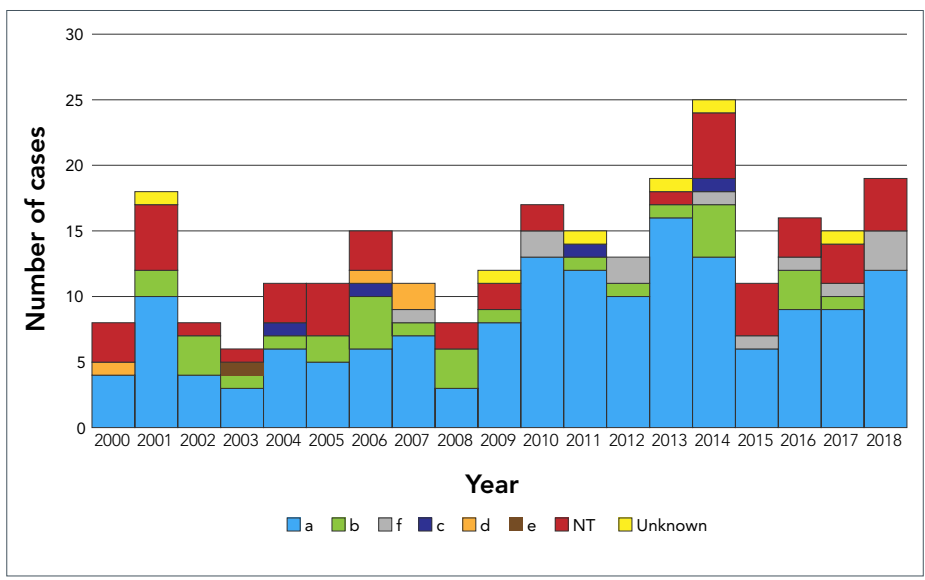

Abbreviations: $a$, invasive Haemophilus influenzae serotype $a$; $b$, invasive Haemophilus influenzae serotype b; $c$, invasive Haemophilus influenzae serotype $c$; $d$, invasive Haemophilus influenzae serotype $d ; f$, invasive Haemophilus influenzae serotype $f$; NT, non-typeable strains

There were no significant changes in Hib rates from 2000 to 2018 $(\beta=-0.01 ; p=0.7)$. Of the $29 \mathrm{Hib}$ cases, three were adults and 26 children aged three years and younger. Of the 29 Hib cases, 14 (48\%) were age-appropriately vaccinated, 10 (35\%) were either unvaccinated or not up-to-date with their vaccinations, three $(10 \%)$ were age ineligible for vaccination and two $(7 \%)$ had unknown vaccine history.

Of the 246 cases with information on clinical manifestation, bacteremia ( $n=201 ; 81.7 \%)$ and pneumonia $(n=87 ; 35.4 \%)$ were the most commonly reported manifestations. Of the 258 cases of $\mathrm{Hi}, 222(86.0 \%)$ were hospitalized. Of the 226 cases with outcome information, 19 deaths (8.4\%) were reported. Of these 19 cases, 11 were serotype a, five were non-typeable, two were serotype $f$ and one was serotype $b$.

No significant difference was observed in the hospitalization and fatality rates of males and females (Table 1).

\section{Invasive meningococcal disease}

There was no significant change in the incidence rate of IMD (per 100,000 population) from 2000 to $2018(\beta=0.03 ; p=0.3$ ). The incidence rate (per 100,000 population) was the highest for 
infants younger than one year old (29.57; Cl: 17.83-49.04) and children one to four years old (3.99; Cl: 2.00-7.98).

Of the 31 cases of IMD, 16 cases (52\%) were serogroup B, seven (23\%) were serogroup W, four (13\%) were serogroup C, one $(3 \%)$ was serogroup $Y$ and three $(10 \%)$ were of unknown serogroup (Figure 5). The seven serogroup W cases all occurred between 2012 to 2018, constituting 47\% of all IMD cases during that period. No serogroup W cases were reported prior to 2012.

Figure 5: Distribution of invasive meningococcal disease cases, by year and serogroup, northern Canada, 2000-2018

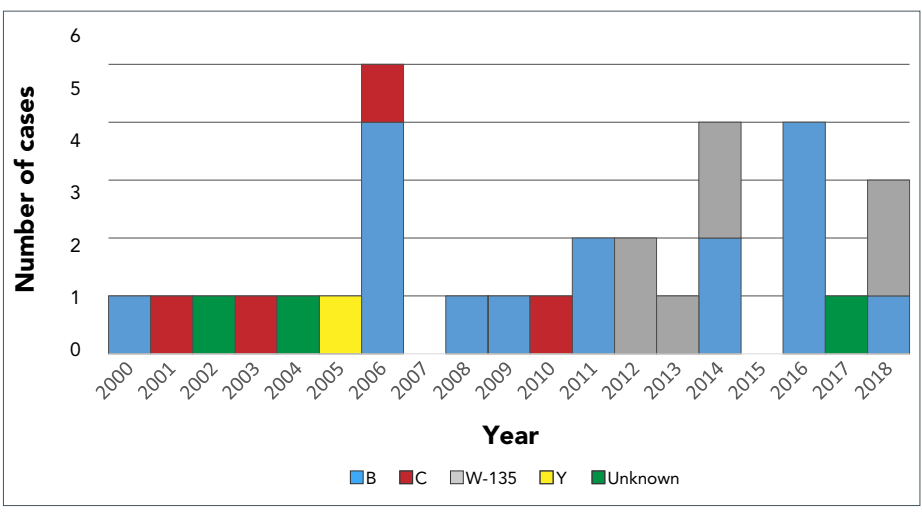

Abbreviations: $B$, invasive meningococcal disease serotype $B ; C$, invasive meningococcal disease serotype $C ; W$, invasive meningococcal disease serotype $W ; Y$, invasive meningococcal disease serotype $Y$

The median age of the serogroup $B$ cases was younger than one year, with a range of $0-30$ years; the median age of the serogroup $\mathrm{W}$ cases was younger than one year, with a range of $0-28$ years; and the median age of the serogroup $C$ cases was 29.5 years, with a range of $0-56$ years.

The difference in the average annual incidence rates between those of Indigenous and non-Indigenous origin was not significant (Table 1). Of the 30 cases with information on clinical manifestation, meningitis $(n=21 ; 70 \%)$ and bacteremia $(n=13$; $43 \%$ ) were most commonly reported and $90 \%$ were hospitalized. Of the 30 cases with fatality information, four deaths (CFR: 13\%) were reported.

No significant difference was observed in the hospitalization and fatality rates of males and females (Table 1).

\section{Invasive group B streptococcal disease}

There was no significant change in the incidence rate of GBS from 2000 to $2018(\beta=-0.01 ; p=0.6)$. The incidence rate (per 100,000 population) was highest for infants younger than one year (46.16; Cl: 29.09-73.26) and adults 60 years and older (3.97; $\mathrm{Cl}: 2.07-7.64)$. The difference in the average annual incidence rates between those of Indigenous and non-Indigenous origin was not significant (Table 1).
Of the 44 GBS cases, 16 cases (36\%) were neonatal and 28 cases (64\%) were non-neonatal. Of the 16 neonatal cases, 10 were early onset disease, five were late onset disease and one was unknown. Of the seven neonatal cases with serotype information, four cases were serotype III and the remaining three were serotype $\mathrm{la}, \mathrm{lb}$ and $\mathrm{V}$. Of the 15 neonatal cases with information on clinical manifestation, bacteremia $(n=10)$ and meningitis $(n=6)$ were most commonly reported.

Of the 28 non-neonatal cases, the median age was 55.5 years (range 0-88 years). Of the 25 non-neonatal cases with serotype information, 6 (24\%) were serotypes III and V each, three (12\%) were serotypes la, lb and VI each, two (8\%) were serotype IV, and one was serotype II and one serotype VI (4\% each). Of the 27 non-neonatal cases with information on clinical manifestation, bacteremia $(n=21 ; 78 \%)$, septic arthritis $(n=6,22 \%)$ and cellulitis ( $n=5,19 \%)$ were most commonly reported. Of all the non-neonatal cases, $93 \%$ were hospitalized; no deaths were reported.

No significant difference was observed in the hospitalization and fatality rates between males and females (Table 1).

\section{Discussion}

In this report, we describe the epidemiology of invasive bacterial diseases in northern Canada from 1999 to 2018 and compared the incidence rates there to those in the rest of Canada. The rates of most of the invasive disease remained stable over time with the exception of IPD, which trended down, and iGAS, which trended up over time. The incidence of IPD, iGAS and Hib were 2.8, 3.2 and 8.8 times higher, respectively, in northern Canada than in the rest of the country. The average annual incidence rates for IPD, iGAS and $\mathrm{Hi}$ in northern Canada were significantly higher for those who identified as Indigenous than those of nonIndigenous origin. The highest incidence rates were among the very young and older people.

Similar to the Alaskan ICS population (personal communication, T. Zulz, Jan 19, 2021), in northern Canada, IPD presented the highest burden of disease and accounted for over half of the total reported cases during the study period. The incidence of iGAS in northern regions has been increasing, a trend that has also been observed nationally, where the incidence rate has increased three-fold from 2000 to 2018 (34), with outbreaks in the community, military bases and in shelters (35-38). Hia accounted for over $60 \%$ of cases and has been increasing significantly over the years. There is also a high prevalence of Hia in the Alaskan population (personal communication, T. Zulz, Jan 19, 2021). According to data collected from the US Active Bacterial Core surveillance sites, between 2002-2008 and 20092015 , the prevalence of Hia disease increased by $13 \%$ annually with an overall increase of $148 \%$ (39). 
The burden of disease for IMD and GBS is much lower than the other three diseases, and IMD and GBS rates did not significantly change during the study period. The results also indicate that there was no statistical difference between the age-standardized incidence rates of IMD for the rest of Canada and northern Canada.

The rates of IPD have significantly decreased over the years due to a significant decrease in PCV7 and PCV10-specific vaccine serotypes, indicating that the conjugate vaccine programs have been successful at reducing the circulation of the serotypes these vaccines protect against. There were no significant decreases of PCV13-specific serotypes over the years. Further study is required to monitor the impact of the PCV13 vaccine versus the PCV10 vaccine, especially following the recent switch from the PCV13 vaccine schedule to a mixed PCV10 and PCV13 vaccine schedule in the Québec regions.

Non-vaccine serotypes have significantly increased following the introduction of the conjugate vaccine programs. PPV23-specific serotypes have also increased and the high percentage of breakthrough disease indicates that the PPV23 vaccine is not as effective at preventing disease in the 65-year-and-older ICS population compared to the conjugate vaccines in the younger ICS population.

Rates of Hib have not significantly changed in northern Canada during this study period. No IMD serogroup $C$ cases have been reported since 2010 and this may be due to the implementation of the Men-C-C vaccine in routine childhood vaccine programs by the mid-2000s (40). Within the last seven years, serogroup $B$ and $\mathrm{W}$ have been reported exclusively and the highest burden has been in infants younger than one year.

\section{Strengths and limitations}

The ICS program is an important source of epidemiological and laboratory-linked information. It is the only enhanced invasive bacterial disease surveillance system that provides information on ethnicity in Canada, which means that it can be used to monitor the epidemiology of invasive bacterial diseases among the Indigenous peoples of northern Canada.

ICS is a passive surveillance system and some cases may be missed. In some communities, antibiotics may have been started prior to the collection of cultures. Results are unstable due to the small number of cases and small population sizes; therefore, caution should be used when interpreting the results. Because of incomplete reporting of clinical manifestation, vaccine history and underlying conditions and risk factors, these results may be under or overestimated. In addition, not all cases had further serotyping done.

\section{Conclusion}

The burden of invasive bacterial diseases is higher in the northern ICS population than the rest of Canada, especially in the Indigenous population. We have limited health information for the large Indigenous population in northern Canada, but existing health disparities need to be monitored and addressed. Ongoing surveillance is needed to continue monitoring disease trends, support prevention and control strategies, and inform immunization recommendations.

\section{Authors' statement}

$\mathrm{GH}$ - Methodology, software, formal analysis, investigation, data curation, writing-original draft, writing-review and editing, visualization

IM - Methodology, formal analysis, writing-original draft, writing-review and editing

RST - Methodology, formal analysis, writing-original draft, writing-review and editing

WHD - Methodology, formal analysis, writing-original draft, writing-review and editing

GJT - Methodology, formal analysis, writing-original draft, writing-review and editing

YAL - Methodology, formal analysis, writing-review, editing and project administration

$C D$ - Conceptualization, writing-review and editing

FR-D - Methodology and writing-review and editing

SGS - Conceptualization, methodology, writing-review and editing, project administration

\section{Competing interests}

None.

\section{Acknowledgements}

We would like to thank all members of the Canadian International Circumpolar Surveillance Invasive Bacterial Diseases Working Group for their invaluable contribution to the International Circumpolar Surveillance.

\section{Funding}

Canada's participation in the International Circumpolar surveillance was paid for by the Public Health Agency of Canada.

\section{References}

1. Parkinson AJ, Bruce MG, Zulz T, Parkinson A; International Circumpolar Surveillance Steering Committee. International Circumpolar Surveillance, an Arctic network for the surveillance of infectious diseases. Emerg Infect Dis 2008;14(1):18-24. DOI PubMed

2. Vaudry W, Talling D. Invasive pneumococcal infection in first nations children in northern Alberta. Can Commun Dis Rep 2002;28(20):165-72. PubMed 
3. Christiansen J, Paulsen P, Ladefoged K. Invasive pneumococcal disease in Greenland. Int J Circumpolar Health 2004;63 Suppl 2:214-8. DOI PubMed

4. Singleton R, Hammitt L, Hennessy T, Bulkow L, DeByle C, Parkinson A, Cottle TE, Peters H, Butler JC. The Alaska Haemophilus influenzae type $b$ experience: lessons in controlling a vaccine-preventable disease. Pediatrics 2006;118(2):e421-9. DOl PubMed

5. Degani N, Navarro C, Deeks SL, Lovgren M. Invasive bacterial diseases in northern Canada. Emerg Infect Dis 2008;14(1):34-40. DOI PubMed

6. Bruce MG, Deeks SL, Zulz T, Navarro C, Palacios C, Case C, Hemsley C, Hennessy T, Corriveau A, Larke B, Sobel I, Lovgren M, Debyle C, Tsang R, Parkinson AJ. Epidemiology of Haemophilus influenzae serotype a, North American Arctic, 2000-2005. Emerg Infect Dis 2008;14(1):48-55. DOI PubMed

7. Li YA, Martin I, Tsang R, Squires SG, Demczuk W, Desai S. Invasive bacterial diseases in northern Canada, 2006-2013. Can Commun Dis Rep 2016;42(4):74-80. DOI PubMed

8. Statistics Canada. Data tables, 2016 Census. Catalogue no. 98-400-X2016156. Ottawa (ON): Statistics Canada; (accessed 2020-01-31). https://www12.statcan.gc.ca/censusrecensement/2016/dp-pd/dt-td/Rp-eng.cfm?TABID=2\&La $\mathrm{ng}=\mathrm{E} \& A P A T H=3 \& D E T A I L=0 \& D I M=0 \& F L=A \& F R E E=0 \& G$ $C=0 \& G I D=1257309 \& G K=0 \& G R P=1 \& P I D=110511 \& P R I D=$ 10\&PTYPE $=109445 \& S=0 \& S H O W A L L=0 \& S U B=0 \&$ Tempor $\mathrm{al}=2017 \& \mathrm{THEME}=122 \& \mathrm{VID}=0 \& \mathrm{VNAMEE}=\& \mathrm{VNAMEF}=\& \mathrm{D} 1$ $=0 \& D 2=0 \& D 3=0 \& D 4=0 \& D 5=0 \& D 6=0$

9. Public Health Agency of Canada. Canadian immunization guide [Internet]. Ottawa (ON): PHAC; 2020 (accessed 2020-06-30). https://www.canada.ca/en/public-health/ services/canadian-immunization-guide/introduction.html

10. National Advisory Committee on Immunization (NACl). Update on pediatric invasive pneumococcal disease and recommended use of conjugate pneumococcal vaccines. Can Commun Dis Rep 2010;36(ACS-3):1-30. https://www. canada.ca/content/dam/phac-aspc/migration/phac-aspc/ publicat/ccdr-rmtc/10pdf/36-acs-3.pdf

11. Papadatou I, Spoulou V. Pneumococcal vaccination in high-risk individuals: are we doing it right? Clin Vaccine Immunol 2016;23(5):388-95. DOl PubMed

12. Government of Newfoundland and Labrador. Newfoundland and Labrador immunization manual: section 2: routine immunization schedules. St John's (NL): Government of Newfoundland and Labrador; 2019.

13. Government of Newfoundland and Labrador. Newfoundland and Labrador immunization manual: section 5: immunization programs for high risk groups. St John's (NL): Government of Newfoundland and Labrador; 2019.

14. Government of Yukon. Yukon immunization program: section 8 - biological products. Whitehorse (YU): Government of Yukon; 2019.
15. Government of Northwest Territories. NWT immunization schedule: publicly funded vaccines for infants and children. Yellowknife (NT): Government of Northwest Territories; 2018.

16. Chief Public Health Office. Routine immunization schedule 2010: adults previously immunized. Iqaluit (NU): Government of Nunavut; 2010.

17. Government of Quebec. Pneumococcal polysaccharide vaccine. Québec (QC): Government of Quebec; 2017 (accessed 2020-05-31). https://www.quebec.ca/en/ health/advice-and-prevention/vaccination/pneumococcalpolysaccharide-vaccine

18. Public Health Agency of Canada. Case definitions for diseases under national surveillance. Can Commun Dis Rep 2009;35 Suppl 2:1-123. https://www.canada.ca/content/ dam/phac-aspc/migration/phac-aspc/publicat/ccdrrmtc/09pdf/35s2-eng.pdf

19. Austrian R. The quellung reaction, a neglected microbiologic technique. Mt Sinai J Med 1976;43(6):699-709. PubMed

20. Tyrrell GJ, Lovgren M, Kress B, Grimsrud K. Invasive group A streptococcal disease in Alberta, Canada (2000 to 2002). J Clin Microbiol 2005;43(4):1678-83. DOI PubMed

21. Altschul SF, Gish W, Miller W, Myers EW, Lipman DJ. Basic local alignment search tool. J Mol Biol 1990;215(3):403-10. DOI PubMed

22. Centers for Disease Control and Prevention. Streptococcus Laboratory. Protocol for emm typing. Atlanta (GA): CDC; 2018 (accessed 2020-03-31). https://www.cdc.gov/streplab/ groupa-strep/emm-typing-protocol.html

23. Slotved HC, Elliott J, Thompson T, Konradsen HB. Latex assay for serotyping of group B Streptococcus isolates. J Clin Microbiol 2003;41(9):4445-7. DOI PubMed

24. Ledeboer NA, Doern GV. Haemophilus. In: Versalovic J, Carroll KC, Jorgensen JH, Funke G, Landry ML, Warnock DW, editors. Manual of clinical microbiology. $10^{\text {th }}$ ed. Washington (DC): ASM Press; 2011. p. 585-602.

25. Falla TJ, Crook DW, Brophy LN, Maskell D, Kroll JS, Moxon ER. PCR for capsular typing of Haemophilus influenzae. J Clin Microbiol 1994;32(10):2382-6. DOl PubMed

26. Riou JY, Guibourdenche M. Laboratory methods: Neisseria and Branhamella. Paris (FR): Institut Pasteur; 1992.

27. Clinical Laboratory Standards Institute. CLSI M100-S26: Performance standards for antimicrobial susceptibility testing, 26 $6^{\text {th }}$ ed. Wayne (PA): CLSI; 2016.

28. Statistics Canada. Aboriginal peoples of Canada: highlight tables, 2001 Census. Tables: 97F0024X2001007. Ottawa (ON): Statistics Canada; (accessed 2020-01-31). https://www150.statcan.gc.ca/n1/en/ catalogue/97F0024X2001007 
29. Statistics Canada. 2011 National Household Survey: data tables. Catalogue number: 99-011-X2011034. Ottawa (ON): Statistics Canada; (updated 2016-01-07; accessed 2020-01-31). https://www12.statcan.gc.ca/nhsenm/2011/dp-pd/dt-td/Ap-eng.cfm?LANG=E\&APATH=3 \&DETAIL $=0 \& D I M=0 \& F L=A \& F R E E=0 \& G C=0 \& G I D=0 \& G K$ $=0 \& G R P=0 \& P I D=107504 \& P R I D=0 \& P T Y P E=105277 \& S=0$ $\&$ SHOWALL $=0 \& S U B=0 \&$ Temporal $=2013 \&$ THEME $=94 \& \mathrm{~V}$ $\mathrm{ID}=0 \& \mathrm{VNAMEE}=\& \mathrm{VNAMEF}=$

30. Statistics Canada. 2006 Census area profiles of population. Profile of Aboriginal Peoples for Canada, Provinces, Territories, Census Divisions and Census Subdivisions, 2006 Census. 2006 Census of Population, Statistics Canada Catalogue no. 94-578-XCB2006001. Ottawa (ON): Statistics Canada; (updated 2015-02-09; accessed 2020-01-31). https://www12.statcan.gc.ca/datasets/Index-eng.cfm?Temp oral $=2006 \&$ Theme $=73 \& V N A M E E=\& G A=-1 \& S=0$

31. Statistics Canada. Data tables, 1996 Census. Catalogue no. 95F0251XDB96001. Ottawa (ON): Statistics Canada; (updated 2019-06-04; accessed 2020-01-31). https://www12. statcan.gc.ca/English/census96/data/tables/Rp-eng.cfm?LA $\mathrm{NG}=\mathrm{E} \& A \mathrm{PATH}=3 \& \mathrm{DETAIL}=1 \& \mathrm{DIM}=0 \& \mathrm{FL}=\mathrm{A} \& \mathrm{FREE}=1 \& \mathrm{GC}$ $=0 \& G I D=0 \& G K=0 \& G R P=1 \& P I D=15504 \& P R I D=0 \& P T Y P E=8$ $9103 \& S=0 \& S H O W A L L=$ No\&SUB=0\&Temporal $=2006 \& \mathrm{THE}$ $\mathrm{ME}=7 \& \mathrm{VID}=0 \& \mathrm{VNAMEE}=\& \mathrm{VNAMEF}=$

32. Centre for Population Health Data. 2011 Population Weights. Ottawa (ON): Statistics Canada; 2020.

33. Fay MP, Feuer EJ. Confidence intervals for directly standardized rates: a method based on the gamma distribution. Stat Med 1997;16(7):791-801. PubMed

34. Public Health Agency of Canada. Notifiable disease pre-built charts. Ottawa (ON): PHAC; 2021 (accessed 2021-02-28). https://diseases.canada.ca/notifiable/charts-pre-built
35. Loewen K, Bocking N, Matsumoto CL, Kirlew M, Kelly L. Epidemiologic features of invasive group A Streptococcus infection in a rural hospital: 6-year retrospective report and literature review. Can J Rural Med 2017;22(4):131-8. PubMed

36. Athey TB, Teatero S, Sieswerda LE, Gubbay JB, Marchand-Austin A, Li A, Wasserscheid J, Dewar K, McGeer A, Williams D, Fittipaldi N. High incidence of invasive group A Streptococcus disease caused by strains of uncommon emm types in Thunder Bay, Ontario, Canada. J Clin Microbiol 2016;54(1):83-92. DOI PubMed

37. Hammond-Collins K, Strauss B, Barnes K, Demczuk W, Domingo MC, Lamontagne MC, Lu D, Martin I, Tepper M. Group A Streptococcus Outbreak in a Canadian Armed Forces Training Facility. Mil Med 2019;184(3-4):e197-204. DOI PubMed

38. Dickson C. 2021 International Circumpolar Surveillance Invasive Bacterial Disease Working Group meeting. Updating public health guidelines for invasive group A streptococcus. Forthcoming, 2021.

39. Soeters HM, Blain A, Pondo T, Doman B, Farley MM, Harrison LH, Lynfield R, Miller L, Petit S, Reingold A, Schaffner W, Thomas A, Zansky SM, Wang X, Briere EC. Current epidemiology and trends in invasive Haemophilus influenzae disease-United States, 2009-2015. Clin Infect Dis 2018;67(6):881-9. DOI PubMed

40. National Advisory Committee on Immunization (NACI). Meningococcal $C$ conjugate vaccination recommendations for infants. An Advisory Committee Statement (ACS). Can Commun Dis Rep 2007;33(ACS-11):1-12. https://www. canada.ca/en/public-health/services/reports-publications/ canada-communicable-disease-report-ccdr/monthlyissue/2007-33/preamble.html 